\title{
Variational and nonvariational principles in quantum transport calculations
}

\author{
Zhongqin Yang, ${ }^{1}$ Alan Tackett, ${ }^{2}$ and Massimiliano Di Ventra ${ }^{1}$ \\ ${ }^{1}$ Department of Physics, Virginia Polytechnic Institute and State University, Blacksburg, Virginia 24061 \\ ${ }^{2}$ Department of Physics and Astronomy, Vanderbilt University, Nashville, Tennessee 37235
}

(Received 8 April 2002; published 16 July 2002)

\begin{abstract}
A variational principle is not generally satisfied in steady-state quantum transport as opposed to the case of ground-state problems. We show that for a short-range potential, a functional for the scattering amplitude can be introduced that is stationary for arbitrary variations about the exact scattering wave function. However, except for the special case of spherically symmetric potentials, the functional does not satisfy any minimum principle even in linear response and for single-channel scattering. The absence of a minimum principle puts severe limitations on the choice of trial wave functions in transport calculations. Examples of electronic transport in selected quantum wires will be presented to illustrate the problem.
\end{abstract}

DOI: 10.1103/PhysRevB.66.041405

PACS number(s): 73.40.Jn, 73.40.Cg, 73.40.Gk, 85.65.+h

The variational method is a powerful tool to estimate the ground-state energy of a particle with Hamiltonian $H{ }^{1}$ The method relies on the use of a trial wave function $\chi$ (or a family of trial wave functions) which reproduces as closely as possible the actual ground-state wave function $\psi_{G S}$. The functional

$$
E[\chi]=\frac{\langle\chi|H| \chi\rangle}{\langle\chi \mid \chi\rangle}
$$

has then the property that

$$
E[\chi] \geqslant E_{G S}=\left\langle\psi_{G S}|H| \psi_{G S}\right\rangle .
$$

If the trial wave function $\chi$ depends on certain parameters, then due to Eq. (2), the best estimate for the ground-state wave function and consequently the ground-state energy can be obtained by minimizing the functional [Eq. (1)] with respect to these parameters. Total-energy calculations in condensed-matter physics, for instance, are based on such a principle. ${ }^{2}$ The families of trial wave functions used in actual calculations vary from linear combinations of atomic orbitals to plane waves, and the choice of one family with respect to another depends mainly on numerical convenience. ${ }^{2}$ In all choices, however, confidence in the convergence of the results with respect to the best estimate of the ground-state wave function is guaranteed by the existence of the variational principle [Eq. (2)].

Electronic transport in a given sample connected to external reservoirs is neither an equilibrium nor a ground-state problem. The physical observable that one needs to calculate in this case is the electric current across the sample. In general, therefore, one should not expect to have a minimum principle from which the best estimate of the scattering amplitudes can be obtained. Note that the absence of such a principle puts severe limitations on the choice of trial wave functions in transport calculations since a minimum principle is necessary to uniquely determine the best estimate from a family of trial wave functions. ${ }^{3}$ This problem was recognized a long time ago. ${ }^{4}$ It was demonstrated ${ }^{4}$ that a minimum principle still applies to the scattering amplitudes for a spherically symmetric potential at zero energy for the very special case when $(i)$ a single $s$-wave channel contributes to scatter- ing at that energy and (ii) the potential does not support bound states or, if it does, the radial part of the Hamiltonian has a negative expectation value on the bound states-trial wave functions. ${ }^{4}$

In recent years, electric-current calculations have received renewed attention in view of the remarkable experimental progress in nanoscale electronics. ${ }^{5}$ In particular, computational methods are being developed that address fundamental issues in quantum transport in nanoscale structures from first principles. ${ }^{6-13}$ These types of calculations are shedding new light on the microscopic mechanisms of electronic transport in nanoscale devices. ${ }^{910,12-15}$ These methods rely on either the self-consistent solution of the Lippman-Schwinger equation $^{6-9}$ or the Keldish nonequilibrium Green's function formalism. ${ }^{10-13}$ In the noninteracting quasiparticle approximation, both approaches are equivalent. In this type of calculation, the basis set used to represent the scattering-wave functions ranges, as in usual total-energy calculations, from localized basis functions to plane waves. ${ }^{6-13}$ The choice of one basis set with respect to the other depends on numerical convenience. However, unlike the case of total-energy calculations and for the reasons described above, caution has to be used in choosing a given family of trial wave functions.

In this paper we show that for any short-range potential, a functional for the scattering amplitude can be introduced that is stationary for arbitrary variations about the exact scattering-wave function. Except for spherically symmetric potentials, however, the functional does not satisfy any minimum principle. In this case, the calculation of the current could thus show large errors if completeness of the basis set is not properly checked. We will illustrate the problem with selected examples of transport in quantum wires using plane waves as the basis set.

Our starting point is the integral Lippman-Schwinger equation $^{6,7}$ that can be written for each scattering energy in operator notation as

$$
|\Psi\rangle=\left|\Psi^{0}\right\rangle+G^{0} V|\Psi\rangle
$$

where $G^{0}$ and $\left|\Psi^{0}\right\rangle$ are the Green's function and wave function, respectively, of the system in the absence of the scat- 
tering potential $V$. The potential $V$ is assumed to tend asymptotically to zero more rapidly than $1 / r{ }^{7}$ The wave function $\left|\Psi^{0}\right\rangle$ satisfies usual scattering boundary conditions. ${ }^{7,16} \mathrm{We}$ now introduce a functional for the scattering amplitude. The latter is proportional to $\mathrm{t}^{3,17}$

$$
f=\left\langle\Psi^{0}|V| \Psi\right\rangle .
$$

For a given energy and for single-channel scattering the current is simply proportional to the scattering amplitude. Under these conditions then, stationarity of the scattering amplitude implies stationarity of the current. We introduce the following functional

$$
A[\chi]=\frac{\left|\left\langle\Psi^{0}|V| \chi\right\rangle\right|^{2}}{\left\langle\chi\left|V-V G^{0} V\right| \chi\right\rangle} .
$$

We first show that if $\chi=c \Psi$, where $c$ is a proportionality constant, then $A[\chi]=f$, i.e., it is proportional to the exact scattering amplitude. Indeed, the wave function $\chi$ satisfies the Lippman-Schwinger equation ${ }^{3}$

$$
|\chi\rangle=c\left|\Psi^{0}\right\rangle+G^{0} V|\chi\rangle .
$$

The denominator of Eq. (5) therefore reduces to $c^{2}\left\langle\Psi^{0}|V| \Psi\right\rangle$, which exactly cancels a factor from the numerator to give $A[\chi]=f$.

We are now left to demonstrate that for any arbitrary variation of $\chi$ of the type

$$
|\chi\rangle=c|\Psi\rangle+|\delta \chi\rangle
$$

the functional [Eq. (5)] is stationary. To first order in the variation $\delta \chi$, the variation $\delta A$ is

$$
\delta A=2\left\langle\delta \chi|V| \Psi^{0}\right\rangle-\left\langle\delta \chi\left|V-V G^{0} V\right| \Psi\right\rangle .
$$

The variation $\delta A$ can be zero for arbitrary $\delta \chi$ if and only if

$$
|g\rangle=2 V\left|\Psi^{0}\right\rangle-\left(V-V G^{0} V\right)|\Psi\rangle
$$

is zero. This implies

$$
\left(1-G^{0} V\right)|\Psi\rangle=b\left|\Psi^{0}\right\rangle,
$$

where $b$ is a multiplicative constant. The last equation is the Lippman-Schwinger equation [Eq. (3)] for a generic exact solution $b \Psi$ of the scattering problem.

For a spherically symmetric potential the functional [Eq. (5)] can be decomposed in partial-wave contributions, each of which is proportional to the functional introduced by Schwinger. ${ }^{4}$ In this case a minimum principle is satisfied for single- $s$-wave-channel scattering and no bound states. ${ }^{4,18}$ In general, however, the functional [Eq. (5)] does not satisfy either a minimum or a maximum principle.

We now illustrate the implications of these findings for actual calculations. We restrict ourselves to the study of selected atomic wires. A schematic of one such wire is represented in Fig. 1(a), where a sample is sandwiched between two metal electrodes that we model with ideal metals (jellium model). ${ }^{6,7}$ The two electrodes are kept at a finite bias $V_{B}$ (see Fig. 1(b), where we have assumed the left-hand electrode is positively biased). The electron wave functions are (a)

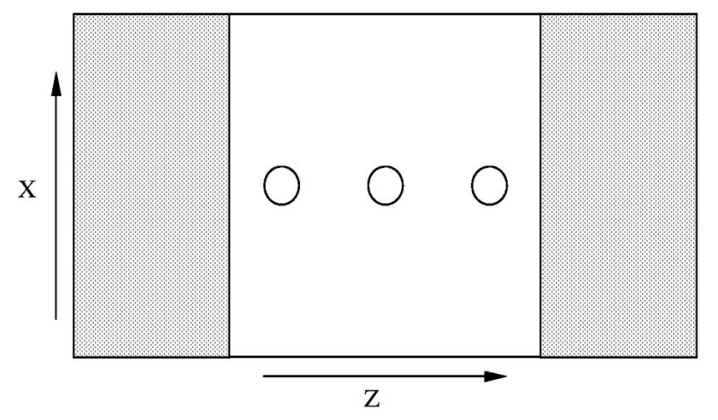

(b)

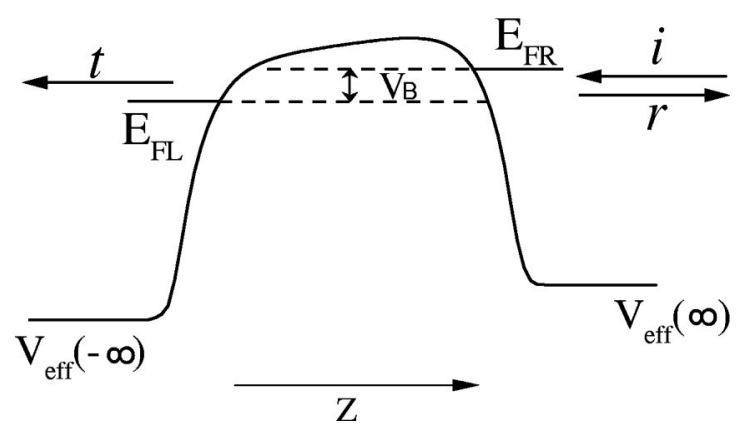

FIG. 1. (a) Schematic of one of the systems under investigation. Three atoms are sandwiched between semi-infinite bulk electrodes. (b) Schematic of the total effective potential of the bare electrodes with bias $V_{B}$. The left electrode is positively biased. Electrons incident from the right electrode are partly transmitted into the left electrode with probability $t$, and partly reflected back into the right electrode with probability $r$.

computed by solving the Lippman-Schwinger equation [Eq. (3)] iteratively to self-consistency in steady state for the continuum part of the spectrum. ${ }^{6,7}$ The bound states, if any, are calculated by direct diagonalization of the total Hamiltonian. ${ }^{7}$ Exchange and correlation are included in the density-functional formalism within the local-density approximation. ${ }^{19}$ The current at zero temperature is computed from the wave functions $|\psi\rangle$ of the electrode-molecule system by integrating the energy region between the right and left Fermi levels [see Fig. 1(b)]. The continuum region between the bottom of the left-hand electrode [indicated as $v_{\text {eff }}(-\infty)$ in Fig. 1(b)] and the right Fermi level has been divided into 40 energy points and convergence has been checked by increasing the number of energy points.

For each scattering energy, we are now faced with the choice of the trial wave functions to represent the best estimate of the exact scattering solution. We choose plane waves to represent the wave functions. ${ }^{7}$ Apart from other technical advantages, like, e.g., the fact that the Green's function $G^{0}$ and the potential $V$ can be analytically written in this basis set, or the absence of Pulay-like forces in the calculation of current-induced forces, ${ }^{7,8}$ plane waves form a complete set in any Hilbert space, and convergence of both scattering amplitudes and electric current can be easily checked by simply increasing their number in the family of trial wave functions. 


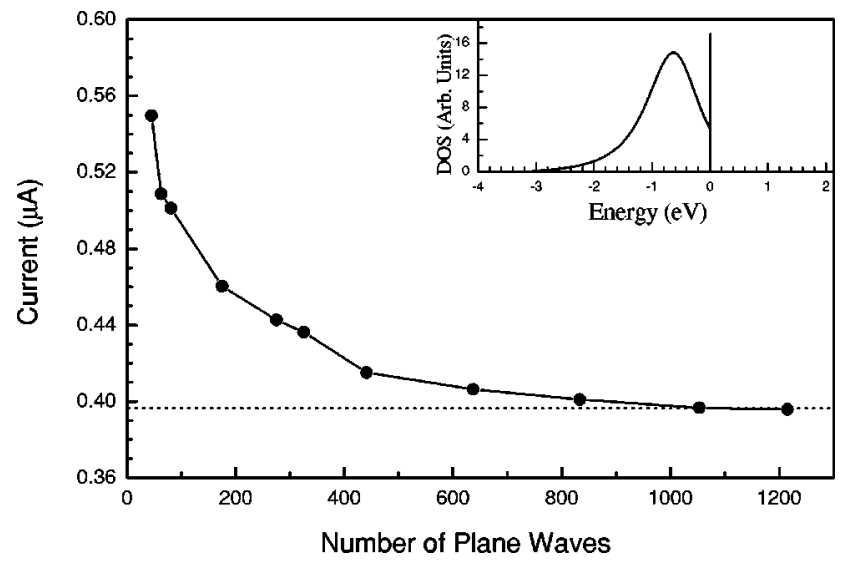

FIG. 2. Current vs number of plane waves for one $\mathrm{H}$ atom sandwiched between two bulk electrodes. The bias is $0.01 \mathrm{~V}$. The inset shows the density of states as a function of energy. The zero energy value is taken as the left Fermi level.

We stress here that any other basis set could be used in principle provided that convergence with increasing number of basis functions be checked. In the examples below, for each total number of plane waves the total charge density of the system (sum of the charge density from both the continuum and discrete part of the spectrum ${ }^{7}$ ) has been converged to less than $1 \times 10^{-3} \%$ for each plane-wave component.

In Fig. 2 we plot the electric current as a function of the number of plane waves for a single hydrogen atom between two metal electrodes. The distance of the hydrogen atom from the electrodes is 4 a.u. ${ }^{20}$ The external bias is $V_{B}$ $=0.01 \mathrm{~V}$ (linear-response regime) and the $s$ orbital of the hydrogen atom is mostly responsible for transport in this system (corresponding to the peak of the density of states in the inset of Fig. 2). Furthermore, the scattering potential has almost spherical symmetry in the region of interest, and the total Hamiltonian does not support any bound state. The physical situation then corresponds to the one for which the functional [Eq. (5)] satisfies a minimum principle. This is clearly evident in Fig. 2 where the current for a fixed number of plane waves (or equivalently the scattering amplitude in this linear-regime case) is always larger than the (asymptotically) converged current (dotted line in Fig. 2).

As we have shown in this paper, for a general short-range potential a minimum principle is not guaranteed. This case is illustrated in Fig. 3 where we plot the electric current as a function of the number of plane waves for three $\mathrm{Si}$ atoms between metal electrodes. The external potential is $V_{B}$

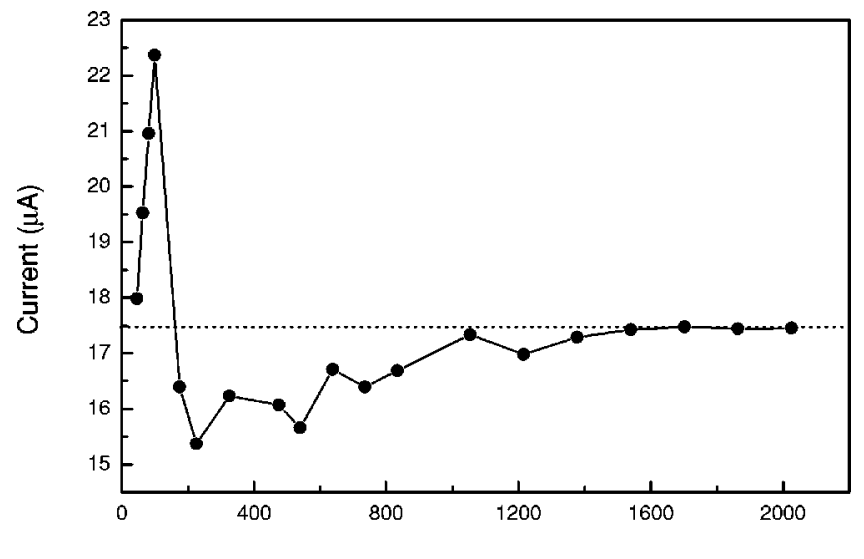

Number of Plane Waves

FIG. 3. Current vs number of plane waves for three Si atoms sandwiched between two bulk electrodes. The bias is $0.1 \mathrm{~V}$.

$=0.1 \mathrm{~V}$. The Si atomic positions are kept equal to their relaxed value at zero bias: the Si-Si distances are 4.2 a.u., and the left and right $\mathrm{Si}$ atoms are at 2.1 a.u. from the electrodes [see Fig. 1(a)]. It is evident from Fig. 3 that the current for different values of the number of plane waves can be either larger or smaller than the (asymptotically) converged current (dotted line in Fig. 3). Furthermore, convergence with the number of plane waves is reached in an oscillatory way. It is also evident from this figure that relative errors of as much as $30 \%$ in the value of the current can be obtained with a fixed number of basis functions.

In summary, we have shown that for any short-range potential, a functional for the scattering amplitude can be introduced that is stationary for arbitrary variations about the exact scattering-wave function. The functional, however, does not satisfy any minimum principle. This puts severe limitations in the choice of trial wave functions in transport calculations. In particular, this study shows that a fixed number of basis functions is not generally enough to assure convergence in electric current calculations.

This work is supported in part by the National Science Foundation Grants No. DMR-01-02277 and DMR-01-33075, Carilion Biomedical Institute, and Oak Ridge Associated Universities. Acknowledgement is also made to the Donors of The Petroleum Research Fund, administered by the American Chemical Society, for partial support of this research. The calculations reported in this paper were performed on the beowulf cluster of the Laboratory for Advanced Scientific Computing and Applications at Virginia Tech.
${ }^{1}$ A. Messiah, Quantum Mechanics (Dover, New York, 1999), p. 763.

${ }^{2}$ See, e.g., M.C. Payne, M.P. Teter, D.C. Allan, T.A. Arias, and J.D. Joannopoulos, Rev. Mod. Phys. 64, 1045 (1992).

${ }^{3}$ See, e.g., J. R. Taylor, Scattering Theory (J. Wiley \& Sons, New York, 1972).

${ }^{4}$ See, e.g., J. Schwinger, Phys. Rev. 72, 742 (1947); W. Kohn, ibid. 74, 1763 (1948).
${ }^{5}$ See, e.g., Molecular Electronics: Science and Technology, edited by A. Aviram and M. A. Ratner (New York Academy of Sciences, New York, 1998).

${ }^{6}$ N.D. Lang, Phys. Rev. B 52, 5335 (1995).

${ }^{7}$ M. Di Ventra and N.D. Lang, Phys. Rev. B 65, 045402 (2002).

${ }^{8}$ M. Di Ventra and S.T. Pantelides, Phys. Rev. B 61, 16207 (2000).

${ }^{9}$ N. Kobayashi, M. Brandbyge, and M. Tsukada, Phys. Rev. B 62 , 8430 (2000). 
${ }^{10}$ J. Taylor, H. Guo, and J. Wang, Phys. Rev. B 63, 245407 (2001).

${ }^{11}$ P.S. Damle, A.W. Ghosh, and S. Datta, Phys. Rev. B 64, 201403 (2001).

${ }^{12}$ M. Buongiorno Nardelli, J.-L. Fattebert, and J. Bernholc, Phys. Rev. B 64, 245423 (2001).

${ }^{13}$ H.J. Choi and J. Ihm, Phys. Rev. B 59, 2267 (1999).

${ }^{14}$ M. Di Ventra, S.T. Pantelides, and N.D. Lang, Phys. Rev. Lett. 84, 979 (2000); Appl. Phys. Lett. 76, 3448 (2000); M. Di Ventra, S.-G. Kim, S.T. Pantelides, and N.D. Lang, Phys. Rev. Lett. 86, 288 (2001); M. Di Ventra, S.T. Pantelides, and N.D. Lang, Phys. Rev. Lett. 88, 046801 (2002).

${ }^{15}$ Y. Xue, S. Datta, and M.A. Ratner, J. Chem. Phys. 115, 4292 (2001).

${ }^{16}$ A. Messiah, Quantum Mechanics (Dover, New York, 1999), p. 801.
${ }^{17}$ Note that this expression for the scattering amplitude is valid only when the $T$ matrix $\left(T=V+V G^{0} T\right)$ can be approximated (first Born approximation) with the value of the potential $V$, i.e., for high energies and/or weak potentials.

${ }^{18}$ For this demonstration we refer the reader to the original references (see Refs. 3 and 4).

${ }^{19}$ P. Hohenberg and W. Kohn, Phys. Rev. B 136, 864 (1964); W. Kohn and L.J. Sham, Phys. Rev. 140, A1133 (1965). The exchange-correlation functional used in this work is the one of D.M. Ceperley and B.J. Alder, Phys. Rev. Lett. 45, 566 (1980), as parametrized by J.P. Perdew and A. Zunger, Phys. Rev. B 23, 5048 (1981).

${ }^{20}$ Note that this distance does not correspond to the equilibrium position of a $\mathrm{H}$ atom between two metal electrodes. 